\title{
Photonics and Web Engineering 2011
}

\author{
Ryszard S. Romaniuk
}

\begin{abstract}
The paper presents a digest of chosen technical work results shown by young researchers from different technical universities in this country during the SPIE-IEEE Wilga 2011 symposium on Photonics and Web Engineering. Topical tracks of the symposium embraced, among others, nanomaterials and nanotechnologies for photonics and telecom, sensory and nonlinear optical fibers, object oriented design of hardware, photonic metrology, optoelectronics and photonics applications, photonicselectronics co-design, optoelectronic and electronic systems for telecom, astronomy and high energy physics experiments, JET and pi-of-the sky experiments development. The symposium is an annual summary in the development of numerable Ph.D. theses carried out in this country in the area of advanced electronic and photonic systems. It is also an occasion for young researchers to meet together in a large group (under the patronage of IEEE) spanning the whole country with guests from this part of Europe. A digest of Wilga references is presented [1-92].
\end{abstract}

Keywords-nanomaterials, optical fibers, optoelectronics, photonics, measurement systems, optical communication systems, photonic sources and detectors.

\section{INTRODUCTION}

T HE 28th Symposium of young scientists WILGA 2011 on Photonics and Web Engineering has gathered over 150 participants. There were presented around 100 papers mainly concerning the realized $\mathrm{Ph} . \mathrm{D}$. theses and participation in research projects relevant to the topical area of the meeting. There were also presented a few plenary papers introducing the audience into new research areas of photonics and electronics. The symposium is organized under the auspices of the SPIE - The International Society for Optical Engineering, IEEE (Poland Section and Region 8), Photonics Society of Poland, KEiT PAN, PKOpto SEP and WEiTI PW. The symposium is organized annually by young researchers from the PERG/ELHEP Laboratory of ISE PW with cooperation of SPIE and IEEE Student Branches. Media patronage over the symposium is extended by Elektronika monthly technical magazine. Symposium proceedings are published by Elektronika, JET - Journal of Electronics and Telecommunications KEiT PAN and Proceedings SPIE. Wilga Symposium is topically associated with the cyclic research meetings on optical fibers and their applications organized in Bialowieza (Bialystok Uni. Technology) and Krasnobrod (UMCS Univ., Lublin) every 18 months. Below, there are shown some presentations from the main of the most interesting sessions or topical tracks of WILGA 2011 Symposium.

\section{TOPICAL TRACKS OF WILGA 2011}

The topical session and tracks of WILGA 2011 were as follows: nanotechnologies and nanomaterials for optoelectronics

R. S. Romaniuk is with Warsaw University of Technology, Poland (e-mail: rrom@ise.pw.edu.pl). and photonics, optical fibers for sensors and all-photonic devices for sensors, active optical fibers, sensors and sensory networks, object oriented design of optoelectronic and photonic hardware, photonics applications, advanced bioelectronics and bioinformatics, co-design of hybrid photonic - electronic systems, computational intelligence in optoelectronics and robotics, development in the wide-angle astronomic observations of the whole sky - pi-of-the-sky project, processing and imaging of multimedia data streams, machine vision, vehicles - quadrocopter and Mars rover, analog transmission systems in noisy conditions with digital reverse transmission channel, optoelectronic and photonic metrology, reconfigurable measurement systems, high performance - low-jitter lowlatency transmission systems - White Rabbit, thermonuclear fusion experiments - JET and ITER, research results update from HEP experiments - TOTEM and CMS/LHC in CERN. A number of Wilga sessions concerned applications aspects of photonic and electronic circuits and systems, including in this advanced applications which combine hardware and software. A separate session track was organized by SPIE, IEEE, OSA and PSP - Photonics Society of Poland students for the new students beginning their adventure with the science of photonics and electronics.

\section{NANOMATERIALS FOR PHOTONICS AND OPTICAL COMMUNICATIONS}

It is predicted that a considerable role in photonics and electronics may play composite nanomaterials using palladium or nickel doped fullerenes like $\mathrm{C}_{60}: \mathrm{Pd}$. Such materials are manufactured by physical or chemical deposition from the gas phase (PVD-CVD) and vaporization away of the side-by products like hydrogen. Such a material has amorphous mixed structure consisting of fullerene and graphite-like phases. Applying approximation methods for effective material, it is possible to determine the complex value of the refractive index of the composite. The fullerene-palladium layers of very developed surface $\left(>100 \mathrm{~m}^{2} / \mathrm{g}\right)$ are researched for applications as very effective hydrogen sensors. There are used for the measurements, the resistance and dielectric permittivity changes of these layers under the influence of absorption and desorption of the gas.

Carbonaceous materials as nano-tubes, nano-filaments, nano-layers and nano-crystallites diamond, graphite, diamondlike, fullerene, heterostructure carbon layers are used in the research on the new field emitters. Such emitters have potentially large efficiency and emission stability, low power consumption, as well as long life time. They are referred to as environmentally friendly. The constructions of carbon based field emitters embrace the following: flat displays, CRT, microwave lamps, X-ray lamps, fluorescent lamps with cold 
cathode. The PVD technology is used for manufacturing of these lamps, where the sources of the deposited materials are $\mathrm{C}_{60}$ and $\mathrm{Ni}$.

Nano-composites are also manufactured by doping the nanocrystallites with active ions like $\mathrm{Pr}^{3+}$ and positioning these nanostructures in the polymer matrix. The active ions are isolated from the polymer matrix. The matrix, most commonly made of PMMA and PDMS, provides good optical and mechanical properties, is easy machining and low price. There were manufactured nanocomposites in IMiO PW Laboratory of the following components - PDMS-YSZPr and PMMA-Y-Pr. The materials were measured using a wideband spectroscope techniques. The nanocomposites keep their luminescent properties of the input nano-powders. There is added a wideband luminescence of the polymer matrix. The effective isolation of the active ions from the polymer matrix allows for application of these materials in active components of polymer photonics.

\section{Optoelectronic AND Electronic COMPONENTS}

Alternating current electroluminescent lamps ACEL are components which emit visible light. They may be manufactured by a cheap printing technology on the polymer substrate in the form of large-area components. These features facilitate their prospective applications in the lighting technology of the future. The basic problem is ruggedness of these components and their lifetime. The lighting efficiency is measured as a function of time as well as their lifetime and degradation. The measured parameters are luminance, emitted wavelength work frequency, nominal voltage, component capacity, dependence of the wavelength on the frequency, other degradation effects. Increase in the work frequency (from 50 to $200 \mathrm{~Hz}$ or more) causes a shift in the emitted light to longer wavelengths. The researched ACELs consist of a polymer foil substrate with deposited transparent electrode from $\mathrm{In}_{2} \mathrm{O}_{3}: \mathrm{SnO}_{2}$, on which there is superimposed the basic electroluminescent component - electroluminescent layer $\mathrm{ZnS}: \mathrm{Cu}, \mathrm{Cl}$, isolator $\mathrm{BaTiO}_{3}$ and metal electrode $\mathrm{Ag}$. The work is carried in IMiO PW laboratory.

ADC circuits are subject to many requirements to increase the working rate and resolution, decreasing the footprint, lowering the consumed power and price. Intelligent, cyclic ADC integrated circuits are subject to research in order to be applied in them highly efficient multidimensional, adaptive estimation algorithms. In each cycle, the successive estimates of the input signal samples are calculated as words of a constant length. Each sample of the input signal is converted independently in $k$ cycles. In other words, code formation in the digital part is exchanged by code calculation in a form of long bit words of a constant length, which is bigger than the final resolution of the converter. This allows for signal description in both parts of the circuit - analog and digital, by continuous variables and for introduction of an analytical conversion quality criterion. Application of multidimensional estimation algorithms allows for concurrent estimation of the value of sample of the input signal and of interfering factors external and internal (interference, drifts). This in turn allows for on-line elimination of these interferences on the conversion quality. In the classical CADC, the problems of overloading is solved by lowering the gain. In the ICADC, bigger gain and optimal conversion algorithms lead to increase of the ENOB parameter (standard IEEE 1241). The simulation tools allow for estimation of the necessary cycles in order to obtain the required value of ENOB. Implementation of ICADC does not require any changes in the architecture of the analog part of the circuit. The modification of the digital part is however required. The prototypes of ACADC were manufactured using CMOS 350nm technology and are investigated by the research group at ISE PW.

\section{Optical Fiber Technology}

Liquid crystal photonic optical fibers are birefringent and nonlinear photonic components. Manufacturing of a LCPCF bases on filling with a liquid crystal the micro pores of the structural material building a classical PCF. They may guide a beam of light by refractive and photonic mechanism. What more, the switching between these guiding mechanisms depends on the relative values of the ordinary and extraordinary refractions of the LC as well as a relation of the latter values to the refraction of the host glass. LCPCF are subject of research for sensory applications and for complex processing of optical information. The LCPCF fibers of complex internal structure are analyzed by methods of the effective refractive index, distribution to base functions, and numerically. There were analyzed theoretically and experimentally LCPCF filled with nematic LC. The molecules of the LC inside the fiber were reoriented. There were tested the following orientations: planar, radial and axial. The following orientations are possible for planar setting: longitudinal (along the fiber axis) and transversal (perpendicular to the fiber axis), as well as angular (intermediate positioning, with LC molecules oriented at particular angle to the fiber axis). Material anisotropy, and due to this, strong coupling between the $\mathrm{E}$ and $\mathrm{H}$ fields (which could not be omitted as in scalar case) requires formulation of vector fields instead of scalar ones. The results of calculations lead to modal fields for photonic propagation in nematic LCPCF. E-M fields are determined for various values of above mentioned refractive indices. The sensitivities of the fibers depend on the thermal dispersion of these indices. Spectral, thermal and mechanical stress characteristics were measured in these fibers for various LC molecule orientations and phase transitions in the LC.

The B777 airplane has $12 \%$ of composites in its construction. The newest B787 has 50\%. Composite materials, working under changing stress have to be monitored on-line. A measurement network of optical fiber Bragg gratings was integrated inside the wings of a small sport airplane Aero AT3. Similar experiments are carried out in optical fiber laboratory at Faculty of Physics WUT. Optical fiber Bragg gratings and highly birefringent fibers were integrated inside a composite beam. The beam was subject to cyclic thermal $\left(-40^{\circ} \mathrm{C}\right.$ to $+110^{\circ} \mathrm{C}$ ), moisture and mechanical load. Thermal sensitivity of the FBG was determined as $11-13 \mathrm{pm} / \mathrm{K}$. The sensing net is of hybrid nature Bragg and polarimetric to measure 
concurrently thermal and dynamic mechanical properties of the composite beam. There were researched the influence of lamination processes on the sensor net characteristics. The influence of the birefringent fiber orientation in the laminate on the sensor performance was determined. The laminate and sensor aging processes were observed. There were researched also bow-tie fibers and HB PCF ones. Sensitivity of the fibers was enhanced by optimized direct covering which gave better mechanical coupling between the glass and composite laminate.

Plastic optical fibers are a cheap alternative to solve the access problem to the optical LAN. Access with a fiber directly to the subscriber's home is of essentials meaning and is called the last mile problem. It enables providing a considerable bandwidth to the subscriber, of the order $10 \mathrm{~Gb} / \mathrm{s}$. Such a bandwidth is required, according to today's estimates to serve all needs of a larger, nondependent household. These needs may embrace: safety, VoIP, VOD, IPTV, 3DHDTV, VC, Internet, intelligent home information, measurement and control network. One of the alternatives to the classical glass optical fibers is application of single mode, large mode area, plastic micro structural fibers with photonic mode of beam transmission mPOF. mPOFs allow for wide shaping of the transmission parameters like modal field, mode distribution, wide extent of singlemode propagation (thus providing wide bandwidth), decreased macro-bending losses (via strongly bound photonic propagation), and acceptable spectral transmission losses (only one $\mathrm{km}$ of fiber is statistically needed). The advantages of mPOF, from the point of view of the user, ore good mechanical properties, and the possibility to reconfigure the network by the user himself. Other advantage is low price of the cables and active components. Technical advantages are: potentially very broad region of single mode transmission, very large area of the fundamental mode, low macro-bending losses, easier possibility to tailor the dispersion and polarization parameters of the fiber. The work on MPOF are carried out at ITME and IMiO PW laboratories.

Tellurite glass optical fibers doped with active erbium ions are applied for building lasers with the up-conversion light generation mechanism. They emit green light. Short wavelength fiber laser is optically pumped with cheap laser diodes emitting 980 and 1480nm. The host material for fiber lasers may be ZBLAN glass or telluride glass. Both glasses have low value of the phonon wave energy. The ZBLAN glasses are less stable chemically, quite susceptible to interaction with water and vapor. Telluride glasses are more resistant to water. The basic composition of telluride glasses for application to optical fibers is as follows: $\mathrm{TeO}_{2}-\mathrm{WO}_{3}-\mathrm{Na}_{2} \mathrm{O}-\mathrm{ZnO}-\mathrm{Nb}_{2} \mathrm{O}_{5}$. The core glass is doped with $\mathrm{Er}$ and has more tellurium oxide at a cost of the zinc oxide. There were drawn optical fibers with cores of $20-35 \mu \mathrm{m}$ in diameters Generation of green light was obtained in short lengths of telluride fibers. A telluride optical fiber laser with up-conversion is under construction. The laser is using an optical fiber loop mirror from one side and FBG mirror from the other. The work is carried out in the IMiO PW photonics laboratory.

Analog transmission systems, performed in arbitrary technology, may work with the transmission rate very close to the
Shannon rate limit for the channel throughput. The condition is an existence of a feed back digital channel of high quality sending the estimates of the signal. The feedback channel should be digital, for example optical fiber. The field of applications of analog transmission systems may embrace: optical fiber LANs, wireless LANs, very high quality LANs, networks working in noisy environments, wireless sensory RFID networks, safety networks.

\section{Biomedical Photonics And Optoelectronics}

Mobile devices, now combine internally many functions like: cellular phone, photo and camera, multimedia player, personal digital assistant, radio and television sets, GPS and GIS, access to Internet, local and remote data bases. There will be added to these the next functions like cloud computing, individual insurance polices (like EHIC - European health insurance card), individual licenses like driving license, wireless credit/payment cards - electronic purse, personal identification, other techniques basing on RFID, but also biomedical measurement system and personal health record cards. Now there are predicted the following health data to be stored first which is combined with the information which may influence the patient therapy, and which includes: data concerning allergy, currently administered and taken medicines, chronic diseases (diabetes, hypertension, epilepsy), actual and historic image records and laboratory results. Such data combined with the patient facilitate many medical procedures, and may be necessary in rescue situations. Current technology and PDA resources allow for realization of many biomedical solutions. The following data acquisition, storing and access parameters and condition are usually determined: quantity of data may be very large, data should be accessible even if the PDA is broken or battery is depleted, thus the carrier should be an SD card. Biomedical data should be well protected against an unauthorized access, like by symmetric cipher with individual SK key. The work on SK for biomedical applications in PDA are carried out in a few research laboratories in this country, among then at WUT. There are considered issues of key distribution and updating, short-term alarm keys, hardware and software requirements for biomedical data mobile stations and servers, and overall system costs.

Low-level laser therapy finds applications for biostimulation and subtle photochemical interactions. Optical power provided by the lighting heads does not overcome $0.5 \mathrm{~W}$ and does not cause thermal effects. Tuning of the wavelength allows for changing of the light penetration depth and for different interaction mechanism with the cellular structures, fibroblasts and oxyhemoglobin, hemoglobin, melanin. Optimal constructions of lighting heads are researched for homogeneous optical power distribution, or programmable lighting for different biomedical applications. The heads are equipped increasingly frequently not in lasers but in LEDs. The work is carried on at PB and WAT laboratories.

Optoelectronics offers many applications for motion and sight disabled persons. Particular attention is paid to a group of devices enhancing the disabled work with the PC. These are different access interfaces, including joysticks fitted in the 
most optimal way to the kind of person's disability, pointers associated with movement of head, feet, or more complex ones associated with eye globe movement, or simpler as optical keyboards. The work on optical keyboard for motion disabled persons of various degree of disability is carried out at PB Optoelectronics laboratory.

Optical fiber capillaries are used to build integrated chemical processors, of Lab on Capillary or LoC type (not to mix with the same abbreviation which stands for Lab on Chip). Optical capillaries allow for simultaneous transmission of light and matter. The light may be transmitted along the fiber in the same direction or in opposite direction as matter. The light may also be transmitted perpendicular to the fiber, or simultaneously both ways to cross the optical power threshold at some place of the capillary reactor. Optical capillary in a LoC system transmits fluid samples of the researched matter, reacting agents, catalytic substances, solvents and light to the reactor point. There are possible measurements in the fluidic mode of LoC work. While the probes and reagents flow through the LoC interaction point, there are performed transmission and dissipation/scattering measurements including turbidimetry, nefelometry and spectral ones. The measuring heads with capillary optical fibers were used for measuring of edible oils quality, fluid fuels, alcohols, but also physiological fluids in the cattle - the latter to determine the fertility periods and diagnose illnesses.

Many calculations of biomedical informatics have data represented as strings. Strings represents DNA and sequences of amino acids. Classification of strings is done using intelligent sorting methods. There are used kernel methods like SVM. The kernel function is interpreted as a measure of similarity. Two biological sequences are considered as similar if they fit well in the SVM classifier. Two graphs are considered as similar when they share many common pathways. Other method to build classifiers bases on mutual information and serves for calculation of orthogonal forward regression. The SVM method was applied to research the sites and classification of albumen phosphorylation. The research concerns the influence on the phosphorylation of new kinds of medicines. Homogeneous classifiers and heterogeneous ones may be implemented in dedicated hardware, in such a way as to increase their usability for biomedical applications. The work is carried out in ISE WUT laboratories, in the research team on Computational Intelligence.

\section{PHOTONIC INTERNET}

A topical track on photonic Internet consisted of papers on photonic LANs, trunk optical lines of ultimate throughput, new optical non-blocking codes, developments in all-optical architectures. One of the concepts, not yet fully confirmed, is application of organic photonics to build the framework of the variety of access networks to the terabit photonic transport network. Micro-structured plastic optical fibers - mPOF are under intense studies, also in this country. They are cheap and are user scalable, even in singlemode version.

\section{Networked Measurement Systems For RESEARCH IN ASTRONOMY}

Wide angle, systematic observations of the whole sky are carried out by research groups from the CFT PAN, IPJ, UW and WUT laboratories. An adequate observational system was designed, manufactured and situated in astronomical observatories. The system consists of ultra-low-noise sCCD cameras and DSP electronics. The pi-of-the-sky research project is realized, which has gathered a lot of own observational data on astronomical objects. These data are correlated with universal data bases. A new observation site has been opened recently in Spain in the INTA institute (Costa de la Cruz). The localization in ESO Las Campanas (LCO) was shifted from the central Chile location to North to SPdA observatory. There was installed a new generation of detectors and cameras of greater sensitivity and considerably lower noises. Positioning of the observational mount consisting of four cameras was done basing on 34 main parameters for the mount and the cameras: absolute orientations HA and DEC against the equatorial set of coordinates (4 parameters), conversion from the counter in the step motor to the values of angles in RA and DEC (2), localization in the rotation space of the axis DEEP/WIDE (8), values of angles between DEEP/DEEP (4), directions of versors CCDx and $\mathrm{CCDz}$ in the space (16). The result of positioning is such a set of 34 parameters for which the difference between the astrometry and the detector is the smallest. A single pixel of the pi-of-the-sky camera is equivalent tp 36 arcsec. Setting angles to HA axis is $4 \mathrm{p}$, DEC axis 27 p, HOME axis $27-37$ arcsec. Thus the mount is very precise. The WIDE mode $\left(40 \times 40^{\circ}\right)$ the cameras observe adjacent areas, and in DEEP mode $\left(20 \times 20^{\circ}\right)$ the fields cover each other.

One of the objects observed by pi-of-the-sky camera are changing stars - Cepheids. There were observed cepheids of the I and II kind, metallic and non-metallic, in the range of magnitudes 6-12, which are subject to contraction and expansion with the first and the second harmonic. There were gathered light curves for more than 150 cepheids. Fourier analysis of the light curves is an efficient tool to classify the cepheids. Over-tone cepheids have sinusoidal shape of the light curves, and classical - a triangular one. There were identified a few cepheids, not yet present in the global catalogues.

The analysis of the observational data of the pi-of-thesky, embracing of more than 1.5 million stars allowed for distinguishing more than 1000 changing stars of different types. There were applied classification criteria from the GCVS and ACVS catalogues. There were discovered around 20 new changing stars and the data on the periods of a few tens of changing stars were more precisely confined.

During the observations in the LCO, during the period 200409 above $1 \mathrm{~TB}$ of reduced data were gathered. The biggest achievement was observation of the burst GRB080319B. The effect was Publisher in Nature 11.09.2008. In January 2011, from the INTA localization, there was observed effect GRB110112B

Measurement data base was prepared for the observation results of spectral characteristics in the vicinity of quasars 
(UMK and PW laboratories). Spectral characteristics contain information concerning wide and narrow absorption lines in the area of dust accretion ring, accretion plasma stream and jets perpendicular to the accretion disc of the black hole. Optimal data organization allows for its 10 time reduction without information loss. To process the measurement data, graphic processors were used. This shortens the data processing of over two orders of magnitude. Development of new calculation technique leads to optimization of data bases and usage of CPU-GPU processor farms.

\section{NuClear Photonics AND Electronics}

Research groups from AGH, WUT-PW, IPJ, UW, IFPiLM, UL and other laboratories participate in the worldwide experiments of the global scale, especially in the domain of high energy physics, nuclear technologies, high-power and high energy lasers, accelerator science, tokamaks and stellarators. One can list the research on: quark-gluon plasma in FAIRGSI Darmstadt in the experiment on compressed baryonic matter, neutrino oscillations in the artificially generated beams, new fundamental particles generated potentially during the hadron collisions in the CMS, ATLAS, TOTEM experiments, thermonuclear synthesis in JET and ITER experiments, superrelativistic astroparticles of energies above $10^{15} \mathrm{eV}$ (ankle) and also over $10^{20} \mathrm{eV}$. These experiments require research on new materials and construction of detectors and accelerators, new measurement methods, algorithms of signal processing, new solutions to photonic and electronic systems fulfilling new requirements.

The CBM experiment is expected to lead to the measurements of a phase diagram for the super-dense nuclear matter and let to determine the phase boundaries between the baryonic matter, quark-gluon plasma and matter super-strongly interacting. Inside the CBM experiment, there are build in this country detector components for silicon tracker. A dedicated ASIC for the tracker is under design and tests, as well as cable connections between the detector and the circuit. A specialized DSP is under design with new processing algorithms for measurement data. The system of detector electronics is optimized against the noise, ruggedness and ionizing radiation hardness. There are carried out simulation calculations of the influence of the series resistance and coupled stray capacitance on the noise parameters of the front end circuit. In the case of a short detector $(2 \mathrm{~cm})$ and long cable $(50 \mathrm{~cm})$ of a decreased crosssection, the noises were lowered, In the case of long detector $(3 \times 3 \mathrm{~cm})$ and short cable $(5 \mathrm{~cm})$ it is necessary to choose a wider metal path of the detector. The issue of power supply and data readout from the two-side detector was solved. A prototype of 16-channel self-triggering readout ASIC, for the silicon stripe detector of the tracker was manufactured. The IC was named TOTO2. The system measures deposited charge and time mark of each collision. The input stage is optimized for long detectors and may work with holes and electrons (two-side detector). Each channel includes a charge sensitive amplifier with a constant discharge current, discriminator with trimming DAC circuit and signal forming circuits.

A digest paper was presented concerning the advances of measurements in the CMS/LHC experiment in CERN. The proton bunches are collided with $7 \mathrm{TeV}$ energy in the mass centre. There is observed increased multiplicity of the collision products. All the effects of the Standard Model were recreated. The search goes on for new physics. A research team from UW and PW has built the RPC Muon Trigger for the CMS experiment. The conceptual and design work goes on a new generation of electronic systems for the sLHC accelerator, InterLock system for the LHC, high performance data distribution for the LHC measurement and control network, photonics and electronics upgrade for the CMS experiment and for TOTEM detector.

The development of thermonuclear experiments JET and ITER basing on tokomaks or stellarator requires application of new photonic and electronic measurement and control systems, as well as safety systems of much bigger measurement and configuration abilities than the ones used today. In a relatively rare plasma, od the temperature bigger than $100 * 10^{6} \mathrm{~K}$, heated ohmically and by microwaves, an exothermic thermonuclear synthesis reaction goes on. In such a reaction, $1 \mathrm{~g}$ of deuterium fuel is equivalent to $11 \mathrm{~T}$ of the coal fuel. The research groups from IFPiLM, UW and PW participate in the work on the Xray spectrometer for measuring the radiation in the vicinity of the tokomak. There are used GEM gas detectors with microholes and electron multiplication mechanism in the avalanche. Multichannel readout electronics basing on the FMC standard, is under construction for these detectors in this thermonuclear experiment.

\section{Multimedia And Telecom Technologies}

The imaging metod with the use of structural light relies on projection of a known image pixel pattern. The 3D scene may be observed with the application of the structural light technology. Depth of the scene is calculated from the distortion of the pattern image. The technique has a number of advantages, as in certain conditions simplicity of calculations, which privilege this method to certain kinds of applications, as imaging of certain static scenes. It has also drawbacks, a small dynamics, eliminating it from other applications, as fast changing scenes. The structural light technique uses de Brujin sequences of the $n$-th order for a chosen alphabet. It is a sequence of signs (here color stripes of structural light) for which each sub-sequence of $n$-signs is unique and appears only once (here the widths of the light stripes and their color). The structural light may be optimized by the choice of stripes colors - RGB, CMY or mixed. The aim of optimization is to minimize the color interferences and maximization of the contrast between the adjacent stripes. The issue is the loss of contrast and color interference after image acquisition. Color segmentation is necessary in separate RGB channels, image analysis in 2D space, calculation of statistical parameters for each window, modification of the color of individual pixel in the adjacent window and image processing off-line. Detection of stripes borders in the structural light is done via the analysis of the value for the hue in the HSV color space.

The future standard of highly efficient video coding H265 is elaborated by the JCT-VC Working Group, which uses the previous standards like VCEG and MPEG. The standard 
should be ready by January 2013. The assumption is that the transmission bit rate would be reduced by $50 \%$ in comparison with the AVC standard while keeping high quality of the image. Typical applications are: VC of low latency, video streaming, broadcasting and multicasting, digital cinema, VoD, large area displays, mobile video, multimedia data acquisition. There are carried out works on coding of the block tree (CTB), prediction unit - internal and external, interpolation process for luma and chroma, image transformations and quantization, smoothing filtration for the predictions and edge transformations, adaptive filter which reduces the distortions in the reconstructed image, entropy coding, context adaptive arithmetic coding, and other components of the video stream processing system.

The coding standard MPEG-4 - AAC uses psychoacoustic model of human hearing system. The AAC set of coder and decoder possesses the perceptual model. The processes of quantizing, de-quantizing, noiseless coding may be realized in a FPGA circuit. The algorithm uses the Huffman code blocks. Application of the psycho - acoustic model relies on the usage of the masking effects to reduce redundant data. The over threshold tones are masked acoustically by strong adjacent acoustic harmonics. For a particular complex sound, there is created a specific masking curve. All tones below the masking curves may be omitted in the coding process, while all others are subject to precise processing in the coder and are further transmitted. The result of the process is data rate reduction, minimization of the distortions, maximization of the sound quality. The parameters of the quality in the psycho acoustic model are: noise coefficient measured as the ratio of noise to the mask (not to the signal floor), level of the mask and its mean value, maximal value of the mask, perceptional measure of the audio quality, objective quality difference. The $\mathrm{AAC}$ algorithms and their modifications are used for the audio signal processing.

Stereovision of large base differs technically in a fundamental way from the systems with a small base. The assumptions to build a stereoscopic image from two distant and nondependent cameras (as differentiated from a single stereoscopic camera with two adjacent lenses for 3D video stream) are: image colors may differ essentially, the cameras may have different parameters, different amplifications, observation angles, scene illumination, geometrical distortions between the views, etc. The aim is to estimate the mutual positioning of the cameras, calculation of the basic image matrix, finding corresponding points - regions, lines of the image, estimation of the image object depths, reconstruction of 3D scene (there may be required more than two views of the scene). Features of the descriptors of image regions, which enable the fusion of the scene are the following: invariance to color and rotation, immunity to geometrical distortions, adaptability for various scales. There are built analytical models concerning the possibility to reduce the transmission rate by elimination the redundant data in the multi-view imaging systems. The system may find applications in generation of 3D images from a few city cameras positioned in a single region with overlapping imaging areas of the individual cameras.

Adaptive methods of motion estimation are parts of the video data processing systems. The methods are implemented in particular hardware solutions, like FPGA circuits of determined resources and confinements.

Estimation of motion is a process associated with comparison of pixels of the current image frame with the reference frame. The aim is to reduce the temporal redundancy between the frames. The most popular method used for motion estimation basing on blocks is BBME. It uses the criterion of block fitting. The H264/AVC protocol uses multiple reference frames and weighted prediction. The applied accuracy is one fourth of a sample. A modification of the method is application of changing estimation block dimension and very fast estimation algorithms. Fast algorithms base on the efficient reduction of searched positions (step search, diamond). They base on the assumption of unimodality of the error surface. They use hierarchical methods multi-resolution, adaptive, and multipath. They utilize simplified fitting criteria. They perform fast and full search and partially remove the distortions. They use mixed techniques to adapt to the movement of the estimated block and fit optimally to the available calculation resources. The work on various aspects of multimedia technologies is done in the laboratory of Multimedia Technology at IRE PW. The achievements of this lab were presented by several young researchers.

\section{KNOWLEDGe Representation}

The issue of knowledge representation, the whole human knowledge, in a formal framework, such that it is possible to realize complex functions, is an intense research field associated with many research disciplines including in this sociology, anthropology, psychology, and from technical sciences information technologies, telecommunications, computer engineering, Internet engineering and others. The current search in the domain of theory and practice of huge data warehoused and data bases are not sufficient. Gathering and generation of knowledge in a mechanistic way is too poor. It seems that a proper approach is rooting the knowledge from fundamental notions - thus in the ontological and metaphysical view. If this is true, it is necessary to create a more or less formal description language for notions, which is not only understandable for computers but also would allow for building an effective foundation for completely new kinds of data bases with internal super or over-correlations, resembling the way of thinking and notion associations of human beings. This language has to be easy for humans and should be written in a script form (if only this is possible). Such a description should, according to the rules of ontology, describe generally the structure of the reality, and derive its concrete instances from the foundations, associated with the notions of existence, and its forms, things and their properties, causality, time, space, necessity and possibility, ability and identity - similarity and modality. Ontological super- data base concerning the human knowledge would include evolutionarily developed, gathered, widened and improved the most general notion designates like: particularity, individuality, a thing, an abstract, general thing, properties, features. The functions realized in the super-base act in the domain of naming particular criteria 
of individual kinds of being and things and ways of their existence. Successively, from such a over- data base there are derived in a proper way and logically particular instances. Gathering the knowledge under the ontological heading is equivalent to collecting, searching, creating, correlating etc. of instances in a fully semantic way and with the use of mechanisms, which we today awkwardly call the AI - artificial intelligence.

Ontological approach starts to find applications in the contemporary ICT and in research on the AI. In the elementary approach, this method is applied to realization of the following processes: categorization, complex sorting, hierarchisation (thus measuring, prediction, functional and non-functional dependencies), and more complex taxonometric (dependencies fully not functional, not quantitative). The work on ontological approach to the super - data bases and communication of these data bases to the outer world is carried out now in many research labs in the world, including this country at UKSW and PW labs.

\section{Photonics And Electronics ApPlications}

A number of session concerning the applications of photonic and electronic circuits and systems included work on particular engineering and technical solutions for various fields like: car industry, airborne industry, robotics, management of the road traffic, remote control methods for utility systems via the Internet, audio and video techniques, biomedicine, safety techniques, home appliances. A group of work concerned the development of a mobile platform for a universal robot equipped with advanced devices like cameras, grippers. Other group concerned the development of distributed measurement networks for minimum energy service of the network of self configuring environmental sensors. These sensors are expected to use a lot of energy harvesting.

\section{Conclusions, ACKNOWledgments, Future}

Photonics and Web Engineering 2011, in its $28^{\text {th }}$ edition keeps the style of being a wide and friendly meeting for young researchers, mainly Ph.D. students. The Wilga Symposium proceedings are good memoirs of the development of photonics, optoelectronics, metrology and some telecom technologies in the academic laboratories in this country.

The author would like to acknowledge all the persons involved in the organization of the conference on 'Photonics and Web Engineering', Wilga 2011, in particular the Program and Organization Committees. The author also would like to thank all participants of Wilga Symposium for making the event again and again a success.

The 2012 Symposia on Photonics and Internet Engineering will be held on 26-29 January at WEiTI PW building in Warsaw and on $28.05-03.06$ in Wilga Resort by PW. The organizers warmly invite young researchers to present their work. The WILGA Symposium web page is under the address: http://wilga.ise.pw.edu.pl.

\section{REFERENCES}

[1] J.R. Just et al., "Highly parallel distributed computing systems with optical interconnections," Microprocessing and Microprogramming, vol. 27, no. 1-5, pp. 489-493, 1989.

[2] A. Dybko et al., "Application of optical fibres in oxidation - reduction titrations," Sensors and Actuators B, vol. 29, no. 1-3, pp. 374-377, 1995.

[3] _ , "Polymer track membranes as a trap support for reagent in fiber optic sensors," Journal of Applied Polymer Science, vol. 59, pp. 719723, 1996.

[4] —, "Efficient reagent immobilization procedure for ion-sensitive optomembranes," Sensors and Actuators B, vol. 34, no. 1, pp. 207-211, 1997.

[5] _ "Assessment of water quality based on multiparameter fiber optic probe," Sensors and Actuators B, vol. 51, pp. 208-213, 1998.

[6] J. Dorosz et al., "Fiber Optic Department of Biaglass," Optica Applicata, vol. 28, no. 4, pp. 267-291, 1998.

[7] — "Multicrucible technology of tailored optical fibers," Optica Applicata, vol. 28, no. 4, pp. 293-322, 1998.

[8] R.S. Romaniuk et al., "Multicore single-mode soft-glass optical fibers," Optica Applicata, vol. 29, no. 1, pp. 15-49, 1999.

[9] R.S. Romaniuk, "Tensile strength of tailored optical fibers," Optoelectronics Review, vol. 8, no. 2, pp. 101-116, 2000.

[10] _ "Manufacturing and Characterization of ring-index optical fibers," Optica Applicata, vol. 31, no. 2, pp. 425-444, 2001.

[11] A. Burd et al., "Pi of the Sky - automated search for fast optical transients over the whole sky," Astronomische Nachrichten, vol. 325, no. $6-8$, p. $674,2004$.

[12] — , "Pi of the Sky - all-sky, real-time search for fast optical transients," New Astronomy, vol. 10, pp. 409-416, 2005.

[13] T. Czarski et al., "Cavity parameters identification for TESLA control system development," Nuclear Instruments and Methods in Physics Research Section A: Accelerators, Spectrometers, Detectors and Associated Equipment, vol. 548, no. 3, pp. 283-297, 2005.

[14] R.S. Romaniuk et al., "Optical network and FPGA/DSP based control system for free electron laser," Bulletin of the Polish Academy of Sciences, vol. 53, no. 2, pp. 123-138, 2005.

[15] T. Czarski et al., "TESLA cavity modelling and digital implementation in FPGA technology for control system development," Nuclear Instruments and Methods in Physics Research Section A: Accelerators, Spectrometers, Detectors and Associated Equipment, vol. 556, no. 2, pp. 565-576, 2006.

[16] _ "Superconducting cavity driving with FPGA controller," Nuclear Instruments and Methods in Physics Research Section A: Accelerators, Spectrometers, Detectors and Associated Equipment, vol. 568, no. 2, pp. 854-862, 2006.

[17] B. Mukherjee et al., "Application of low-cost Gallium Arsenide lightemitting-diodes as kerma dosemeter and fluence monitor for high-energy neutrons," Radiation Protection Dosimetry, vol. 126, no. 1-4, pp. 256260, 2007

[18] W. Ackerman et al., "Operation of a free-electron laser from the extreme ultraviolet to the water window," Nature Photonics, vol. 1, no. 6, pp. 336-342, 2007.

[19] T.R. Wolinski et al., "Photonics Society of Poland established," Metrology and Measurement Systems, vol. 15, no. 2, pp. 241-245, 2008.

[20] R.S. Romaniuk et al., "Metrological aspects of accelerator technology and high energy physics experiments," Measurement Science and Technology, vol. 18, no. 8, p. E01, 2008.

[21] P. Fafara et al., "FPGA-based implementation of a cavity field controller for FLASH and X-FEL," Measurement Science and Technology, vol. 18 no. 8, pp. 2365-2371, 2008.

[22] R.S. Romaniuk, "Capillary optical fiber," Bulletin of the Polish Academy of Sciences, vol. 56, no. 2, pp. 87-102, 2008.

[23] S. Chatrchyan et al., "The CMS experiment at the CERN LHC," Journal of Instrumentation, vol. 3, no. 8, p. S08004, 2008

[24] J. Dorosz et al., "Development of optical fiber technology in Poland," in Proc. SPIE 8010, 2011, pp. 8010-02.

[25] R.S. Romaniuk, "Ultrabroadband photonic Internet," in Proc. SPIE 8010, 2011, pp. 8010-03.

[26] — , "Modulation and multiplexing in ultra-broadband photonic Internet, part I," in Proc. SPIE 8010, 2011, pp. 8010-04.

[27] _ "Modulation and multiplexing in ultra-broadband photonic Internet, part II," in Proc. SPIE 8010, 2011, pp. 8010-05.

[28] B. Niton et al., "Documentation generator for VHDL and MatLab source codes for photonic and electronic systems," in Proc. SPIE 8010, 2011, p. $80100 \mathrm{R}$ 
[29] — "Documentation generator application for VHDL source codes," in Proc. SPIE 8010, 2011, p. 80100S.

[30] — "Documentation generator application for MatLab source codes," in Proc. SPIE 8010, 2011, p. 80100T.

[31] R.S. Romaniuk, "Photonics Letters of Poland - a new peer reviewed Internet publication of the Photonics Society of Poland," Photonics Letters of Poland, vol. 1, no. 1, pp. 1-3, 2009.

[32] — "WILGA Symposium on Photonics Applications," Photonics Letters of Poland, vol. 1, no. 2, pp. 46-48, 2009.

[33] G. Kasprowicz et al., "CCD detectors for wide field optical astronomy," Photonics Letters of Poland, vol. 1, no. 2, pp. 82-84, 2009.

[34] R.S. Romaniuk, "POLFEL - Free Electron Laser in Poland," Photonics Letters of Poland, vol. 1, no. 3, pp. 103-105, 2009.

[35] — " "Modal structure design in refractive capillary optical fibers," Photonics Letters of Poland, vol. 2, no. 1, pp. 22-24, 2010.

[36] - "WILGA Photonics and Web Engineering 2010," Photonics Letters of Poland, vol. 2, no. 2, pp. 55-57, 2010.

[37] _ - "Geometry design in refractive capillary optical fibers," Photonics Letters of Poland, vol. 2, no. 2, pp. 64-66, 2010.

[38] P. Obroślak et al., "Digital techniques for noise reduction in CCD cameras," Photonics Letters of Poland, vol. 2, no. 3, pp. 134-136, 2010

[39] R.S. Romaniuk, "Petabit photonic Internet," Photonics Letters of Poland, vol. 3, no. 2, pp. 91-93, 2011.

[40] S. Chatrchyan et al., "Commissioning of the CMS experiment and the cosmic run at four tesla," Journal of Instrumentation, vol. 5, no. 3, p. T03001, 2010

[41] — "Performance of the CMS Level-1 trigger during commissioning with cosmic ray muons and LHC beams," Journal of Instrumentation, vol. 5 , no. 3 , p. T03002, 2010 .

[42] — "Performance of the CMS drift-tube chamber local trigger with cosmic rays," Journal of Instrumentation, vol. 5, no. 3, p. T03003, 2010

[43] _ _Fine synchronization of the CMS muon drift-tube local trigge using cosmic rays," Journal of Instrumentation, vol. 5, no. 3, p. T03004, 2010.

[44] —, "Commissioning of the CMS High-Level Trigger with cosmic rays," Journal of Instrumentation, vol. 5, no. 3, p. T03005, 2010.

[45] — "CMS data processing workflows during an extended cosmic ray run," Journal of Instrumentation, vol. 5, no. 3, p. T03006, 2010.

[46] — "Commissioning and performance of the CMS pixel tracker with cosmic ray muons," Journal of Instrumentation, vol. 5, no. 3, p. T03007, 2010.

[47] _ _ "Commissioning and performance of the CMS silicon strip tracker with cosmic ray muons," Journal of Instrumentation, vol. 5, no. 3, p. T03008, 2010

[48] — - "Alignment of the CMS silicon tracker during commissioning with cosmic rays," Journal of Instrumentation, vol. 5, no. 3, p. T03009, 2010 .

[49] —, "Performance and operation of the CMS electromagnetic calorimeter," Journal of Instrumentation, vol. 5, no. 3, p. T03010, 2010

[50] — "Measurement of the muon stopping power in lead tungstate," Journal of Instrumentation, vol. 5, no. 3, p. P03007, 2010.

[51] _ _ "Time reconstruction and performance of the CMS electromagnetic calorimeter," Journal of Instrumentation, vol. 5, no. 3, p. T03011, 2010.

[52] — "Performance of the CMS hadron calorimeter with cosmic ray muons and LHC beam data CMS Collaboration," Journal of Instrumentation, vol. 5, no. 3, p. T03012, 2010

[53] — , "Performance of CMS hadron calorimeter timing and synchronization using test beam, cosmic ray, and LHC beam data," Journal of Instrumentation, vol. 5, no. 3, p. T03013, 2010.

[54] _ _ "Identification and filtering of uncharacteristic noise in the CMS hadron calorimeter," Journal of Instrumentation, vol. 5, no. 3, p. T03014, 2010.

[55] — "Performance of the CMS drift tube chambers with cosmic rays," Journal of Instrumentation, vol. 5, no. 3, p. T03015, 2010

[56] — "Calibration of the CMS drift tube chambers and measurement of the drift velocity with cosmic rays," Journal of Instrumentation, vol. 5 , no. 3, p. T03016, 2010.

[57] — "Performance study of the CMS barrel resistive plate chamber with cosmic rays," Journal of Instrumentation, vol. 5, no. 3, p. T03017, 2010.

[58] _ "Performance of the CMS cathode strip chambers with cosmic rays," Journal of Instrumentation, vol. 5, no. 3, p. T03018, 2010.

[59] _ "Aligning the CMS muon chambers with the muon alignment system during an extended cosmic ray run," Journal of Instrumentation, vol. 5 , no. 3, p. T03019, 2010
[60] _ "Alignment of the CMS muon system with cosmic-ray and beamhalo muons," Journal of Instrumentation, vol. 5, no. 3, p. T03020, 2010.

[61] _ _ "Precise mapping of the magnetic field in the CMS barrel yoke using cosmic rays," Journal of Instrumentation, vol. 5, no. 3, p. T03021, 2010.

[62] - "Performance of CMS muon reconstruction in cosmic-ray events," Journal of Instrumentation, vol. 5, no. 3, p. T03022, 2010

[63] A. Burd et al., "Pi of the sky - robotic search for cosmic flashes," in Proc. SPIE 6159, 2006, pp. 154-159.

[64] W.L. Wolinski et al., "Introduction - Laser technology," in Proc. SPIE 6598, 2007, pp. ix-xi

[65] R.S. Romaniuk, "Introduction - Photonics Applications," in Proc. SPIE 6937, 2008, pp. xxix-xli.

[66] G. Kasprowicz et al., "Hardware emulator of the high resolution CCD sensor for the pi-of-the-sky experiment," in Proc. SPIE 6937, 2007, p. 693708.

[67] M. Kwiatkowski et al., "Nios II implementation in CCD camera for Pi of the Sky experiment," in Proc. SPIE 6937, 2007, p. 693709.

[68] A. Brandt et al., "Measurement and control of field in RF GUN at FLASH," in Proc. SPIE 6937, 2007, p. 69370F.

[69] T. Czarski et al., "Multi-cavity complex controller with vector simulator for TESLA technology linear accelerator," in Proc. SPIE 6937, 2007, p. $69370 \mathrm{H}$

[70] P. Strzałkowski et al., "Versatile LLRF platform for FLASH laser," in Proc. SPIE 6937, 2007, p. 69370I

[71] K. Lewandowski et al., "FPGA based PCI mezzanine card with digital interfaces," in Proc. SPIE 6937, 2007, p. 69370J.

[72] L. Dymanowski et al., "Data acquisition module implemented on PCI mezzanine card," in Proc. SPIE 6937, 2007, p. 69370K.

[73] R. Graczyk et al., "FPGA systems development based on universal controller module," in Proc. SPIE 6937, 2007, p. 69370M.

[74] K. Bujnowski et al., "MatLab script to $\mathrm{C}$ code converter for embedded processors of FLASH LLRF control system," in Proc. SPIE 6937, 2007, p. 693700 .

[75] — - "Decomposition of MATLAB script for FPGA implementation of real time simulation algorithms for LLRF system in European XFEL," in Proc. SPIE 6937, 2007, p. 69370P.

[76] R.S. Romaniuk, "Nonlinear glasses and metaglasses for photonics, a review, part I," in Proc. SPIE 6937, 2007, p. 693716.

[77] _ , "Nonlinear glasses and metaglasses for photonics, a review, part II," in Proc. SPIE 6937, 2007, p. 693717.

[78] J. Dorosz et al., "Introduction - Optical Fiber Applications," in Proc. SPIE 7120, 2008, pp. xiii-xv.

[79] M. Kwiatkowski et al., "Advanced camera image data acquisition system for 'Pi-of-the-Sky'," in Proc. SPIE 7124, 2008, p. 7124OF.

[80] A. Kalicki et al., "Ultra-broadband photonic Internet: safety aspects," in Proc. SPIE 7124, 2008, p. 712410.

[81] R.S. Romaniuk, "Introduction - Photonics Applications," in Proc. SPIE 7502, 2009, pp. 7502-01, xxiii-xxiv

[82] _ "Development of free electron laser and accelerator technology in Poland," in Proc. SPIE 7502, 2009, pp. 7502-70.

[83] — , "Institute of Electronic Systems in CARE and EuCARD projects, Accelerator and FEL research, development and applications in Europe,' in Proc. SPIE 7502, 2009, pp. 7502-71.

[84] — "Introduction - Photonics Applications," in Proc. SPIE 7745 2010, pp. 774 501, xiii-xviii.

[85] _ - "Photonics Applications and Web Engineering SPIE-PSP WILGA Symposium series," in Proc. SPIE 7745, 2010, p. 774502.

[86] _ "WILGA Photonics and Web Engineering 2010"" in Proc. SPIE 7745, 2010, p. 774503

[87] J. Modelski et al., "Electronics and telecommunications in Poland, issues and perspectives, part 1," in Proc. SPIE 7745, 2010, p. 774504.

[88] _ - "Electronics and telecommunications in Poland, issues and perspectives, part 2," in Proc. SPIE 7745, 2010, p. 774505.

[89] — "Electronics and telecommunications in Poland, issues and perspectives, part 3," in Proc. SPIE 7745, 2010, p. 774506.

[90] J. Gajda et al., "Development of laser technology in Poland," in Proc. SPIE 7745, 2010, p. 774507

[91] W. Wójcik et al., "Optical fiber technology development in Poland," in Proc. SPIE 7745, 2010, p. 774508 .

[92] R.S. Romaniuk, "EuCARD 2010: European coordination of accelerator research and development," in Proc. SPIE 7745, 2010, p. 774509.

[93] T. Janicki et al., "Integration of multi-interface conversion channel using FPGA for modular photonic network," in Proc. SPIE 7745, 2010, p. $77451 \mathrm{H}$.

[94] A. Zagozdzinska et al., "Parametrized diagnostic module implementation in FPGA structures," in Proc. SPIE 7745, 2010, p. 77451I. 